\title{
REVIEW
}

\section{Emergency department drug therapy for status epilepticus in adults}

\section{A S Lockey}

Status epilepticus is a medical emergency that demands immediate treatment. The purpose of this review is to analyse the different treatment options for drug therapy and determine the most appropriate choice for adults in the emergency department.

S tatus epilepticus is defined as a continuous seizure lasting more than 30 minutes or two or more seizures without full recovery of consciousness between any of them. ${ }^{1}$ The most common and dangerous type is generalised convulsive status epilepticus (GCSE), which is characterised by coma and convulsive movements. The incidence of GCSE in the UK has been said to be 180 to 280 cases per million. ${ }^{2}$ It is a medical emergency and demands immediate treatment as mortality rates of $10 \%$ to $15 \%$ have been reported. ${ }^{3}$

The initial management of the patient in GCSE entails managing the airway, breathing and circulation. While maintaining a clear airway, high flow oxygen is administered. Intravenous access should be obtained and fluid therapy started as necessary. A high priority is the measurement of glucose using a bedside test ("BM"). If this is low, dextrose should be administered intravenously. If the patient has a history of malnutrition or alcoholism, administration of $100 \mathrm{mg}$ thiamine intravenously should be considered.

The emergency physician should then decide which drug to administer as principal therapy to the patient who is still in GCSE. The purpose of this review is to analyse the different options for drug therapy advocated over the years and determine the most appropriate choice for GCSE in adults in the emergency department.

\section{THERAPEUTIC ALTERNATIVES Benzodiazepines}

This group of drugs has traditionally been the first line of treatment for the acute episodes of seizures. They exert their antiepileptic effect by preventing the spread of seizure rather than suppressing the seizure focus. ${ }^{4}$ They have a rapid onset of action due to their high lipid solubility and rapidly penetrate the brain.

\section{Diazepam}

Diazepam (Valium) was described by Gestaut et al in $1965^{5}$ as being "the drug of first choice for the emergency treatment of all cases of status epilepticus", and as such remained unrivalled until relatively recently. It has been reported to halt
GCSE in approximately $80 \%$ of patients in a matter of minutes. ${ }^{6}$ A recognised side effect of intravenous administration of diazepam is venous thrombosis.?

Diazepam is very lipid soluble and also highly lipid bound (up to 99\%), ${ }^{4}$ resulting in a large volume of distribution. It has the longest elimination half life (30 hours) $)^{8}$ of the commonly used benzodiazepines. The drug is rapidly redistributed from the brain to peripheral fat stores despite rapid uptake into the brain, explaining its shorter duration of action (15-30 minutes). Subsequent bolus doses of diazepam for recurrent seizures can therefore lead to accumulation of the drug peripherally. As diazepam has such a long elimination half life, this increases the risks of side effects such as sudden hypotension and respiratory or circulatory collapse. ${ }^{6}$ In addition, diazepam is metabolised to desmethyldiazepam, which has a long half life?

It can be administered intravenously or rectally in the patient with GCSE. Rectal administration of diazepam solution results in peak concentrations within 5 to 30 minutes. ${ }^{9}$ Diazepam therefore continues to be used in the prehospital management of GCSE where intravenous access may be difficult or not an option.

\section{Lorazepam}

Lorazepam is a hydroxylated benzodiazepine, which was originally introduced in the United States as an anxiolytic agent in $1977 .{ }^{10}$ In comparison with diazepam, its redistribution is minimal resulting in therapeutic levels persisting in the brain for a longer period of time. Its anticonvulsant effect is therefore reported as lasting in excess of 24 hours. ${ }^{8}$ These differences are due to a higher percentage of unbound drug. In a study looking at elimination half lives and volumes of distribution in eight healthy volunteers, ${ }^{11}$ the apparent volume of distribution of diazepam was more than 10 times that of lorazepam. Walker ${ }^{12}$ looked at 25 patients treated with lorazepam. All of the nine patients who had GCSE had their seizures terminated within 10 minutes. An additional difference is that lorazepam has no active metabolites.

Lorazepam is not as reliable as diazepam when administered rectally because of slower absorption and variable bioavailability. ${ }^{9}$ It is unsuitable for use in prehospital management, as the intravenous solution needs to be stored in a fridge and there is no reliable rectal preparation. As with diazepam, lorazepam may also cause respiratory depression and hypotension. All benzodiazepines render the patient stuperous making subsequent neurological examination difficult. When compared with diazepam, there is apparently a lesser 
incidence of venous thrombosis after intravenous injection of lorazepam.

\section{Clonazepam}

Clonazepam has also been used in the initial drug management of GCSE and has been compared in clinical trials with diazepam and lorazepam. An intravenous loading dose is used and it has an effect in comparable times. ${ }^{4}$ When compared with lorazepam, ${ }^{13}$ it was felt that although improvement in the EEG was quicker with lorazepam, clinical symptoms resolved more completely with clonazepam.

\section{Midazolam}

Midazolam is a water soluble benzodiazepine with an elimination half life of approximately two hours. ${ }^{14}$ Its effects are similar to diazepam and it has been in common use for minor operative procedures since the mid-1980s because of its anxiolytic and amnesic properties. At body pH, it is highly lipophilic and rapidly enters the brain with a fast onset of action. It has a shorter duration of action than the other benzodiazepines, thus enabling an earlier accurate neurological assessment of the patient. It can subsequently be used as an intravenous infusion. It has been shown in a small study ${ }^{15}$ to be an effective and safe alternative to high dose barbiturates when conventional treatment has failed.

An alternative route of administration for midazolam is the buccal route. This has been shown to be effective in childhood and adolescence with $75 \%$ of episodes ending within 10 minutes of administration. ${ }^{16}$ It was felt to be at least as effective as rectal diazepam in the acute treatment of seizures and, as such, can be considered in the prehospital management of GCSE. Due to its water solubility, midazolam can also be administered via the intramuscular route. ${ }^{17}$ Recently, intranasal midazolam has been shown to be as safe and as effective as intravenous diazepam in the treatment of febrile seizures in children. ${ }^{18}$ More work is needed before it is recognised as an accepted treatment for GCSE in adults.

\section{Phenytoin/fosphenytoin}

Phenytoin was first synthesised in 1908 and its antiepileptic properties were first reported in the 1930s. It is said to control up to $91 \%$ of cases of $\mathrm{GCSE}^{19}$ and readily crosses in to the brain with a slower rate of redistribution than that of the benzodiazepines.

Its use in GCSE is limited by side effects at high infusion rates. At maximal infusion rates, hypotension occurs in $28 \%$ to $50 \%$, and cardiac arrhythmias (bradycardia and ectopic beats) occur in $2 \% .^{20}$ These effects are more common in the elderly and those with pre-existing cardiac disease. It is irritant to tissues and with a pH of 12 is unsuitable for intramuscular administration. It does not, however, depress either respiration or the functioning of the central nervous system in comparison with the benzodiazepines.

The intravenous loading dose should not exceed 50 $\mathrm{mg} / \mathrm{minute}$ and the patient should have full cardiorespiratory monitoring during the infusion. Peak brain concentrations are achieved in three to six minutes ${ }^{2}$ although the anticonvulsant effect may take up to 30 minutes to be apparent. It is because of this that it has been suggested that it is used alongside a benzodiazepine (thus adding a longer acting preparation to the shorter but more rapidly acting benzodiazepine).

A recent addition to the formulary is fosphenytoin, which is a water soluble phosphate ester pro-drug of phenytoin, metabolised to phenytoin in 8 to 15 minutes by endogenous phosphatases. Unlike phenytoin, it is not dissolved in propylene glycol, allegedly partly responsible for side effects such as hypotension and arrhythmias. ${ }^{2}$ It is buffered to a $\mathrm{pH}$ of 8.6 to 9.0 with the result that there is a lower incidence of venous irritation and phlebitis. ${ }^{20}$ It can be administered intravenously or intramuscularly. When administered intramuscularly it produces therapeutic plasma concentrations within 30 minutes. ${ }^{21}$ It has been shown to be as effective as phenytoin in treating GCSE and can be administered at faster infusion rates thus establishing therapeutic concentrations within 10 minutes. ${ }^{22}$ There are however potential side effects with intravenous administration. Worldwide, 21 cases of asystole, ventricular fibrillation or cardiac arrest in association with intravenous fosphenytoin have been reported. ${ }^{23}$ In five of these cases the doses or infusion rates were greater than recommended.

\section{Lignocaine (lidocaine)}

Lignocaine was first used in the treatment of GCSE in $1955 .{ }^{24}$ The exact mechanism of its action is unclear although theories have been postulated regarding its membrane stabilising effects. ${ }^{25}$ Another theory postulated has been that it exerts a central local anaesthetic action on the inhibitory pathway fibres involved in direct cortical stimulation. ${ }^{26}$ The drug is rapidly distributed and has an elimination half life of one to eight hours. ${ }^{27}$

Lignocaine has numerous side effects, ranging from light headedness and drowsiness to cardiovascular collapse and convulsions. The side effects are more likely with the higher infusion rates. Lignocaine has been advocated in GCSE that is uncontrolled by standard benzodiazepine and phenytoin therapy. Phenytoin therapy can be continued during the lignocaine infusion. ${ }^{28}$ It has also been advocated in patients with underlying disease (for example, head injury and COPD) where diazepam induced respiratory depression would be inadvisable. ${ }^{29}$ It is not in common use however because of its side effect profile that can include convulsions.

\section{Barbiturates}

Barbiturates were initially the drug group of choice before the advent of benzodiazepines. They penetrate the CNS rapidly but side effects are quite pronounced such as respiratory depression, coma, and hypotension.

\section{Phenobarbitone}

Phenobarbitone has been shown to be as effective as a combination of diazepam and phenytoin. Shaner $e t a l^{30}$ described a randomised, non-blinded trial involving 36 adult patients presenting to an emergency department with GCSE. They were randomised to receive either diazepam and phenytoin or phenobarbitone. If the patient continued to convulse 10 minutes after starting phenobarbitone, a phenytoin infusion was started.

Outcomes measured included the cumulative convulsion time (total time spent in active convulsive movements) and the response latency (time from the start of treatment to the end of the last convulsion). The cumulative convulsion time was shorter for the phenobarbitone group $(p<0.06)$, as was the response latency $(p<0.10)$. It was concluded that the phenobarbitone regimen is rapidly effective, comparable in safety and is more practical than diazepam and phenytoin.

While this small study seems to suggest a benefit for phenobarbitone, it has been suggested that the depressant effects on respiratory drive, level of consciousness and blood pressure may be detrimental. ${ }^{20}$ It has therefore been suggested that it is only used when combination therapy with benzodiazepines and phenytoin fails.

\section{Pentobarbital and thiopental}

Pentobarbital is given at a loading dose of $5-12 \mathrm{mg} / \mathrm{kg}$ intravenously followed by an infusion in the range $1-10 \mathrm{mg} / \mathrm{kg} / \mathrm{h} .{ }^{31}$ Thiopental is the sulphur analogue of pentobarbital and it is administered at an initial intravenous dose of $4 \mathrm{mg} / \mathrm{kg}$ in order to produce general anaesthesia. The main disadvantages with thiopental are the resultant high risk of hypotension, the high lipid solubility and hence accumulation in fatty tissues, and the presence of an active metabolite (pentobarbital). ${ }^{22}$ 


\section{Others}

Propofol is a phenol derivative, which was first used commercially as a general anaesthetic induction agent in 1986. It is highly lipid soluble and produces induction of anaesthesia at doses of $2-2.5 \mathrm{mg} / \mathrm{kg}$. As with thiopental, it is liable to cause hypotension, but it may also cause profound respiratory depression as well. Concerns with regards to its potential proconvulsant effects seem to be misfounded with myoclonus after its use having previously been misinterpreted as seizure activity. $^{31}$

Valproic acid can be administered via a nasogastric tube or rectally in GCSE. ${ }^{19}$ An intravenous preparation of valproic acid has recently been introduced and has been recommended as a possibility for GCSE partially resistant to diazepam and phenytoin and as a non-depressant alternative to phenobarbital and other CNS depressants. ${ }^{32}$ There has been one case report published ${ }^{33}$ describing severe hypotension when intravenous valproic acid was administered to an 11 year old girl. Further studies are recommended before its use can be fully endorsed.

Paraldehyde and chlormethiazole have also been used in GCSE. Paraldehyde was advocated as far back as $1949^{34}$ as primary treatment for GCSE with initial intramuscular doses followed by intravenous administration. It has substantial toxicity and offers little in comparison to the other drugs available.

Chlormethiazole has gone out of favour because of a variety of side effects including respiratory depression, hypotension, heart block, thrombophlebitis, fever, and severe headache. ${ }^{35} 36$

Corticosteroids are indicated in status epilepticus attributable to mass lesions, arteritis, sickle cell disease or parasitic central nervous system infection.

\section{DRUG TREATMENT OF THE ADULT PATIENT IN GCSE}

A treatment algorithm is suggested (fig l) for the emergency department management of the adult patient in GCSE. This approach is comparable with that recommended in popular texts. ${ }^{37}{ }^{38}$ In contrast, the fourth edition of Clinical Medicine by Kumar and Clark ${ }^{39}$ makes brief mention of the management of GCSE. Diazepam is recommended as the first line drug, followed by phenytoin, and finally general anaesthesia if convulsions persist in excess of 90 minutes. It is hoped that the emergency physician would consider this latter intervention at a much earlier stage if other interventions have been unsuccessful.

\section{Prehospital}

Although this article is not primarily concerned with this area, it would be remiss not to mention prehospital management, as this can affect the emergency department management.

Currently, the mainstay of therapy is the use of diazepam, either rectally by carers and/or ambulance personnel, or intravenously by ambulance personnel. The requirement for lorazepam to be refrigerated makes its use impractical in the prehospital setting.

Studies looking at the use of buccal and intranasal midazolam in the paediatric population ${ }^{16}{ }^{18}$ look promising. In addition, the use of intramuscular midazolam has been explored. ${ }^{17}$ Drugs administered by these routes would be aesthetically more acceptable if their efficacy is shown to be comparable.

\section{Emergency department}

\section{Choice of initial drug therapy}

The choice of first line drug therapy is realistically limited to a combination of benzodiazepines and phenytoin/fosphenytoin. Among the benzodiazepines, diazepam and lorazepam have been the subject of two randomised controlled trials. ${ }^{40}{ }^{41}$

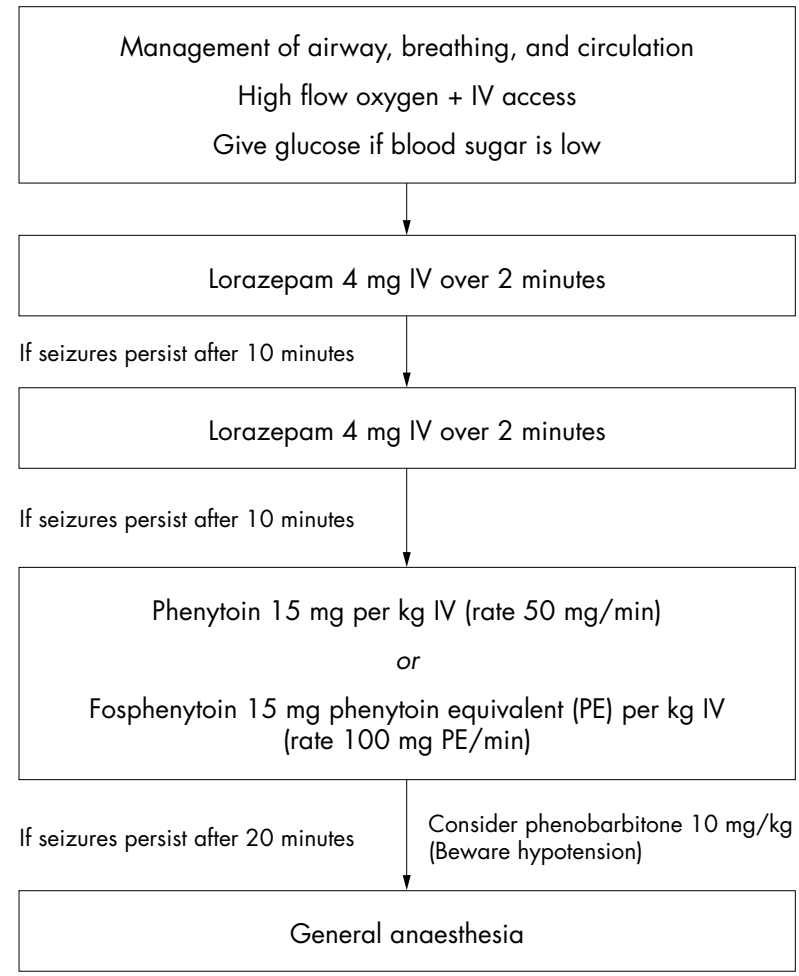

$\begin{array}{ll}\text { General principles: } & \text { Consider thiamine if alcohol misuse is suspected } \\ \text { In refractory GCSE, consider the diagnosis of } \\ \text { pseudostatus } \\ \text { In refractory GCSE, consider the use of other drugs as } \\ \text { local policy dictates }\end{array}$

Figure 1 Treatment algorithm for the emergency department treatment of the adult patients with GCSE.

The landmark study is probably the Veterans Affairs study ${ }^{40}$ which describes a five year, randomised, double blind, multicentre trial looking at four regimens (diazepam/ phenytoin, lorazepam, phenobarbitone, and phenytoin). Altogether 570 adult patients were enrolled in to the study and 518 of them had verified generalised GCSE (no mention is made as to the diagnosis of the other 52 patients). The patients were inpatients at one of 16 Veterans Affairs medical centres or six affiliated university hospitals between 1 July 1990 and 30 June 1995.

A successful outcome was defined by cessation of both clinical and electrical evidence of seizure activity within 20 minutes and no recurrence by an hour after onset of treatment. The success rates for the four regimens were as follows: diazepam/phenytoin $(55.8 \%)$, lorazepam $(64.9 \%)$, phenobarbitone $(58.2 \%)$ and phenytoin $(43.6 \%)$. The only significant difference was between the groups who received lorazepam and phenytoin $(\mathrm{p}=0.002)$. It was concluded that lorazepam is more effective than phenytoin. Although no more efficacious than the other groups, it was felt that lorazepam is easier to use.

Leppik et $a l^{41}$ published a double blind, randomised, controlled trial involving 78 adult patients with 81 episodes of GCSE from three participating centres. A successful outcome was defined by cessation of clinical evidence of seizure activity. The success rates for the two regimens were as follows: lorazepam (one dose $78 \%$, two doses $89 \%$ ), diazepam (one dose $58 \%$, two doses $76 \%$ ). The difference between the two groups was not felt to be significant. The authors were unable to comment on the duration of action of the two study drugs because of the fact that phenytoin had been administered concurrently to most of the patients. It was concluded that lorazepam is at least as effective as diazepam in the treatment of GCSE. 
In both studies, the randomisation processes were either open to question or not entirely clear. In the study by Treiman, treatment kits were placed at four locations within each hospital and the patient was allocated the lowest numbered kit at the site nearest to their location. In the study by Leppik, the manufacturer randomised the assignment of drugs to the kits. There are no details of how the assignment of the kits to the patients was randomised. The other limitation with Leppik's study was that most but not all patients received phenytoin as well. Apart from these issues, both papers stand up well to scrutiny.

In another study published in abstract form, Andermann ${ }^{42}$ compared lorazepam with diazepam in 59 patients with GCSE. Seizure activity was terminated in $82 \%$ of patients after a first dose of $4 \mathrm{mg}$ and $91 \%$ after a second dose in those who had not responded after $10-15$ minutes $(54 \%$ and $84 \%$ with diazepam).

A published abstract by Treiman $e t$ al $^{43}$ described a prospective, randomised, unblinded study of 48 cases. Lorazepam was successful in $76.9 \%$ of the 26 patients receiving it as the first drug. Phenytoin was successful in $54.5 \%$ of patients receiving it as the first drug $(\mathrm{p}<0.05)$.

There are more than 45 papers investigating the issue of first line drug management of GCSE in the form of open, nonrandomised, retrospective trials. Treiman ${ }^{44}$ has analysed the 45 papers published up to 1990 . He stated that control of GCSE was achieved in $79 \%$ of the 1346 patients included with similar results for diazepam, lorazepam and clonazepam (data not published as a review article).

Finally, a frequent misconception is that the side effect profile differs between diazepam and lorazepam. Both drugs have been proven in animal models to reduce the respiratory drive $^{45}$ although no significant difference has been demonstrated in the incidence or severity of this side effect in human studies..$^{3041}$ Hypotension has been noted with diazepam, although significant cases seem to have been associated with associated use of barbiturates. ${ }^{46}{ }^{47}$ Lorazepam has been safely used in high doss (up to $9 \mathrm{mg} / \mathrm{h}$ ) for the treatment of refractory GCSE. ${ }^{48}$ The perceived increased side effects witnessed with diazepam are more likely to be a result of the repeated doses needed to treat GCSE.

In a retrospective study of 1200 adult patients presenting to United Kingdom accident and emergency departments after a seizure published in 1998, Ryan et al ${ }^{49}$ found that in the 59 patients with GCSE the majority (78\%) received diazepam as first line treatment. The evidence suggests that although diazepam is as efficacious as lorazepam at terminating their seizure, we should be considering lorazepam as the first line agent because of its longer duration of action.

Patients with seizures refractory to initial loading with benzodiazepines should be given a loading dose of phenytoin or fosphenytoin. The use of one of these agents should be considered at an early stage, as the anticonvulsant effects will be longer lasting than the benzodiazepines.

\section{Ongoing management}

If the patient is already receiving adequate phenytoin therapy or if phenytoin has no effect, consideration should be given to administering phenobarbitone. The loading dose is $10 \mathrm{mg} / \mathrm{kg}$ (diluted to one in ten with water for injection) and should not exceed a rate of $100 \mathrm{mg} /$ minute. It should be used with caution, as the risks of cardiovascular collapse are higher with the concurrent use of benzodiazepines. It should therefore only be used in a critical care environment.

If the above measures fail to terminate seizure activity within 40 minutes (see fig 1), then induction of general anaesthesia with an appropriate agent and endotracheal intubation should be considered. The discussion relating to the agent used is beyond the scope of this article, but alternatives include barbiturates (for example, thiopental), propofol, and high dose benzodiazepines (for example, midazolam). As all of these agents can result in cardiovascular compromise, the patient should be transferred to an intensive care unit to enable appropriate monitoring and nursing care. Continual EEG monitoring is beneficial as it can enable subclinical seizure activity to be identified.

At all stages, the diagnosis of pseudostatus should be considered in the patient with refractory seizures. Diagnostic features include convulsive episodes lasting longer than 90 seconds, closed eyes during a "tonic-clonic" episode, retained pupillary responses, resistance to eye opening, multiple previous episodes of "status", and a history of other unexplained illnesses and deliberate self harm. ${ }^{50} 51$

\section{CONCLUSION}

In the United Kingdom, patients brought to the emergency department by the ambulance services may have had diazepam administered either intravenously or rectally. Further bolus doses of diazepam will increase levels in the fatty tissues leading to an increased risk of respiratory and CNS depression.

Once intravenous access has been secured, the agent of choice in the emergency department should be lorazepam. The reason for this is because of its prolonged half life and resultant longer duration of action. If lorazepam is ineffective then a loading dose of phenytoin should be started. The newer agent fosphenytoin has recently been advocated as a safe and effective alternative in the emergency initiation and maintenance of anticonvulsive treatment. With its faster infusion rates and shorter onset of action, it has been suggested that it will supercede phenytoin as a complement to benzodiazepines for the early control of seizures. However current reports of adverse events may dent its popularity.

Failure to control convulsions with these measures indicates the need for barbiturates, propofol, or high dose benzodiazepines. Because of the increased risk of cardiovascular instability with these agents, the ideal setting for the ongoing treatment of the patient is in the intensive care unit.

Funding: none

Conflicts of interest: none

\section{REFERENCES}

1 Bone RC. Treatment of convulsive status epilepticus. Recommendations of the Epilepsy Foundation of America's Working Group on Status Epilepticus. JAMA 1993;270:854-9.

2 Delgado-Escueta AV, Fong CY. Status epilepticus: recent trends and prospects. Neurologia 1997;12 (suppl 6):62-73.

3 Lacey DJ. Status epilepticus in children and adults. J Clin Psychiatry 1988;49 (suppl 12):33-6.

4 Treiman DM. Pharmacokinetics and clinical use of benzodiazepines in the management of status epilepticus. Epilepsia 1989;30 (suppl 2):S4-10.

5 Gestaut H, Naquet R, Poite R, et al. Treatment of status epilepticus with diazepam (Valium). Epilepsia 1965;6:167-82.

6 Walker MC, Tong X, Brown S, et al. Comparison of single and repeated dose pharmacokinetics of diazepam. Epilepsia 1998;39:283-9.

7 Hegarty JE, Dundee JW. Sequelae after the intravenous injection of three benzodiazepines - diazepam, lorazepam and flunitrazepam. BM 1977;2:1384-5.

8 Treiman DM. Status Epilepticus. Baillieres Clin Neurol 1996;5:821-39

9 Graves NM. Pharmacokinetics and interactions of antiepileptic drugs. Am J Hosp Pharm 1993;50 (suppl 5):S23-9.

10 Mitchell WG, Crawford TO. Lorazepam is the treatment of choice for status epilepticus. J Epilepsy 1990;3:7-10.

11 Greenblatt DJ, Divoll M. Diazepam versus Lorazepam: relationship of drug distribution to duration of clinical action. Adv Neurol 1983;34:487-92.

12 Walker JE, Homan RW, Vasko MR, et al. Lorazepam in status epilepticus. Ann Neurol 1979;6:207-13.

13 Sorel L, Mechler L, Harmant J. Comparative trial of intravenous lorazepam and clonazepam in status epilepticus. Clin Ther 1981:4:326-36.

14 Galvin GM, Jelinek GA. Midazolam: an effective intravenous agent for seizure control. Arch Emerg Med 1987;4:169-72.

15 Kumar A, Bleck TP. Intravenous midazolam for the treatment of refractory status epilepticus. Crit Care Med 1992;20:483-8.

16 Scott RC, Besag FMC, Neville BGR. Buccal midazolam and rectal diazepam for treatment of prolonged seizures in childhood and adolescence: a randomised trial. Lancet 1999;353:623-6. 
17 Towne AR, DeLorenzo RJ. Use of intramuscular midazolam for status epilepticus. J Emerg Med 1999;17:323-8.

18 Lahat E, Goldman M, Barr J, Bistritzer T, et al. Comparison of intranasal midazolam with intravenous diazepam for treating febrile seizures in children: prospective randomised study. BM 2000;321:83-6.

19 Runge JW. Emergency treatment of status epilepticus. Neurology 1996;46 (suppl 1):S20-3.

20 Lowenstein DH, Alldredge BK. Status epilepticus. N Engl J Med 1998;338:970-6.

21 Uthman BM, Wilder BJ, Ramsay RE. Intramuscular use of fosphenytoin: an overview. Neurology 1996;46 (supp 1):S24-8.

22 Heafield MTE. Managing status epilepticus. BM 2000;320:953-4.

23 Committee on Safety of Medicines/Medicines Control Agency Fosphenytoin sodium (Pro-Epanutin): serious arrhythmias and hypotension. Current Problems in Pharmacovigilance 2000;26:1.

24 Bernhard GC, Bohm E, Hojeberg S. A new treatment of status epilepticus: intravenous injections of a local anaesthetic (lidocaine). Arch Neurol Psychiatry 1955;74:208-14

25 Aggarwal P, Wali JP. Lidocaine in refractory status epilepticus: a forgotten drug in the emergency department. Am J Emerg Med $1993 ; 11: 243-4$

26 Lemmen LJ, Klassen M, Druiser B. Intravenous lidocaine in the treatment of convulsions. JAMA 1978;239:2025.

27 De Giorgio CM, Altman K, Hamilton-Byrd E, et al. Lidocaine in refractory status epilepticus: confirmation of efficacy with continuous EEG monitoring. Epilepsia 1992;33:913-16.

28 Bruni J. Treatment of status epilepticus in adults. Can Med Assoc J 1983;1 28:531-3.

29 Pascual J, Sedano M, Polo JM, et al. Intravenous lidocaine for status epilepticus. Epilepsia 1988;29:584-9.

30 Shaner DM, McCurdy SA, Herring MO, et al. Treatment of status epilepticus: a prospective comparison of diazepam and phenytoin versus phenobarbitol and optional phenytoin. Neurology 1988;38:202-7.

31 Bleck TP. Management approaches to prolonged seizures and status epilepticus. Epilepsia 1999;40 (suppl 1):S59-63.

32 Adin J, Arteaga R, Herranz JL, et al. The use of intravenous valproate. Rev Neurol 1999;29:744-53.

33 White JR, Santos CS. Intravenous valproate associated with significant hypotension in the treatment of status epilepticus. J Child Neurol 1999:14:822-3.

34 Whitty C, Taylor M. Treatment of status epilepticus. Lancet 1949;ii:591-4

35 Lingham S, Bertwistle $\mathrm{H}$, Elliston $\mathrm{HM}$, et al. Problems with intravenous chlormethiazole (heminevrin) in status epilepticus. BM

1980;280:155-6.
36 Browne TR. Paraldehyde, chlormethiazole and lidocaine for treatment of status epilepticus. Adv Neurol 1983;34:509-17

37 Bradley WG, Daroff RB, Fenichel GM, et al. Neurology in clinical practice. London: Butterworth Heinemann, 2000.

38 Sisodiya SM, Sander JW. Loss of Consciousness. Medicine 2000;28:47-51

39 Kumar PJ, Clark ML. Clinical medicine. London: WB Saunders, 1999.

40 Treiman DM, Meyers PD, Walton NY, et al. A comparison of four treatments for generalized convulsive status epilepticus. NEJM 1998;339:792-8.

41 Leppik IE, Derivan AT, Homan RW, et al. Double-blind study of lorazepam and diazepam in status epilepticus. JAMA 1983;249:1452-4

42 Andermann F, Cendes F, Reiher J, et al. A prospective double-blind study of the effects of intravenously administered lorazepam and diazepam in the treatment of status epilepticus. Epilepsia 1992;33 (suppl 3):3

43 Treiman DM, DeGiorgio CM, Ben-Menachem E, et al. Lorazepam versus phenytoin in the treatment of generalized convulsive status epilepticus: report of an ongoing study. [Abstract]. Neurology 1985;35 (suppl 1):284

44 Treiman DM. The role of benzodiazepines in the management of status epilepticus. Neurology 1990;40 (suppl 2):32-42.

45 Terndrup TE, Fordyce WE. Respiratory drive during status epilepticus and its treatment: comparison of diazepam and lorazepam. Epilepsy Res 1995:20:21-30.

46 Prensky AL, Raff MC, Moore M, et al. Intravenous diazepam in the treatment of prolonged seizure activity. N Engl J Med 1967;276:77984

47 Bell DS. Dangers of treatment of status epilepticus with diazepam. BM 1969;1:159-61.

48 Labar DR. High dose intravenous lorazepam for the treatment of refractory status epilepticus. Neurology 1994;44:1400-3.

49 Ryan J, Nash S, Lyndon J. Epilepsy in the A\&E department-developing a code of safe practice for adult patients. J Accid Emerg Med 1998; 15:237-43

50 Howell SJ, Owen L, Chadwick DW. Pseudostatus epilepticus. Q J Med 1989:71:507-19.

51 Reuber M, Enright SM, Goulding PJ. Postoperative pseudostatus: not everything that shakes is epilepsy. Anaesthesia 2000;55:74-8. 